\title{
Thermodynamics of extremal rotating thin shells in an extremal BTZ spacetime and the extremal black hole entropy
}

\author{
José P. S. Lemos, ${ }^{1, *}$ Masato Minamitsuji, ${ }^{1, \dagger}$ and Oleg B. Zaslavskii ${ }^{2}{ }^{\ddagger}$ \\ ${ }^{1}$ Centro Multidisciplinar de Astrofísica - CENTRA, Departamento de Física, \\ Instituto Superior Técnico - IST, Universidade de Lisboa - UL, \\ Avenida Rovisco Pais 1, 1049-001 Lisboa, Portugal \\ ${ }^{2}$ Department of Physics and Technology, Kharkov V. N. Karazin National University, \\ 4 Svoboda Square, Kharkov 61022, Ukraine, and Institute of Mathematics and Mechanics, \\ Kazan Federal University, 18 Kremlyovskaya St., Kazan 420008, Russia
}

(Dated: February 23, 2017)

In a (2+1)-dimensional spacetime with a negative cosmological constant, the thermodynamics and the entropy of an extremal rotating thin shell, i.e., an extremal rotating ring, are investigated. The outer and inner regions with respect to the shell are taken to be the Bañados-Teitelbom-Zanelli (BTZ) spacetime and the vacuum ground state anti-de Sitter (AdS) spacetime, respectively. By applying the first law of thermodynamics to the extremal thin shell, one shows that the entropy of the shell is an arbitrary well-behaved function of the gravitational area $A_{+}$alone, $S=S\left(A_{+}\right)$. When the thin shell approaches its own gravitational radius $r_{+}$and turns into an extremal rotating BTZ black hole, it is found that the entropy of the spacetime remains such a function of $A_{+}$, both when the local temperature of the shell at the gravitational radius is zero and nonzero. It is thus vindicated by this analysis, that extremal black holes, here extremal BTZ black holes, have different properties from the corresponding nonextremal black holes, which have a definite entropy, the Bekenstein-Hawking entropy $S\left(A_{+}\right)=\frac{A_{+}}{4 G}$, where $G$ is the gravitational constant. It is argued that for extremal black holes, in particular for extremal BTZ black holes, one should set $0 \leq S\left(A_{+}\right) \leq \frac{A_{+}}{4 G}$, i.e., the extremal black hole entropy has values in between zero and the maximum Bekenstein-Hawking entropy $\frac{A_{+}}{4 G}$. Thus, rather than having just two entropies for extremal black holes, as previous results have debated, namely, 0 and $\frac{A_{+}}{4 G}$, it is shown here that extremal black holes, in particular extremal BTZ black holes, may have a continuous range of entropies, limited by precisely those two entropies. Surely, the entropy that a particular extremal black hole picks must depend on past processes, notably on how it was formed. A remarkable relation between the third law of thermodynamics and the impossibility for a massive body to reach the velocity of light is also found. In addition, in the procedure, it becomes clear that there are two distinct angular velocities for the shell, the mechanical and thermodynamic angular velocities. We comment on the relationship between these two velocities. In passing, we clarify, for a static spacetime with a thermal shell, the meaning of the Tolman temperature formula at a generic radius and at the shell.

PACS numbers: 04.70.Dy, 04.40.Nr.

Keywords: Quantum aspects of black holes, thermodynamics, three-dimensional black holes, spacetimes with fluids

\section{INTRODUCTION}

The Bañados-Teitelboim-Zanelli (BTZ) spacetime [1] is the spacetime of a (2+1)-dimensional rotating black hole in a negative cosmological constant $\Lambda$ background, being thus an asymptotically anti-de Sitter (AdS) spacetime with length scale $\ell=1 / \sqrt{-\Lambda}$. It obeys a no hair theorem [2], i.e., the black hole is characterized by its gravitational radius $r_{+}$and its Cauchy radius $r_{-}$or, equivalently, by its mass $m$ and its angular momentum $\mathcal{J}$. It is thus a simple $(2+1)$-dimensional spacetime, and as such it provides a way to test and check many different properties of the Kerr black holes in the usual $(3+1)$ -

\footnotetext{
*Electronic address: joselemos@ist.utl.pt

${ }^{\dagger}$ Electronic address: masato.minamitsuji@ist.utl.pt

¥Electronic address: zaslav@ukr.net
}

dimensional spacetime.

An important property of black holes is their entropy, through it one can grasp the microscopic intrinsic elements of a spacetime. For extremal BTZ black holes, i.e., black holes for which the gravitational radius is equal to the Cauchy radius, $r_{+}=r_{-}$, or the angular momentum is equal to the mass, $\mathcal{J}=m \ell$, it was found through topological arguments in the Euclidean sector that the entropy $S$ is [3]

$$
S=0 .
$$

Other studies in string theory [4] suggested that the extremal BTZ black hole entropy is the BekensteinHawking entropy, namely,

$$
S=\frac{A_{+}}{4 G},
$$

where $A_{+}=2 \pi r_{+}$is the event horizon area, actually here a perimeter, $r_{+}$is the gravitational radius, $G$ is the threedimensional gravitational constant, and we use units such 
that the velocity of the light, the Planck constant, and the Boltzmann constant are set to one. Thus, the black hole entropy for extremal BTZ black holes is not a settled issue. On the other hand, for nonextremal BTZ black holes, i.e., black holes for which the angular momentum is less than the mass, $\mathcal{J}<m \ell$, or $r_{+}>r_{-}$, the entropy $S$ is precisely and uniquely given by the Bekenstein-Hawking entropy of Eq. (2). For further studies on the thermodynamics and entropy of BTZ black holes see [5-11].

As the concept of entropy is originally based on quantum properties of matter, it would be useful to study whether the black hole thermodynamics could emerge from thermodynamics of collapsing matter, when we compress matter within its own gravitational radius. So, in order to understand better the physics at the horizon, a promising setting is a thin shell, i.e., a thin ring, in a (2+1)-dimensional spacetime that is compressed quasistatically to its own gravitational radius. Outside the ring, the spacetime has the BTZ form, inside it, the space time is the ground state of the BTZ spacetime, i.e., a zero mass locally AdS spacetime. One can calculate the entropy of this matter ring system for any ring radius $R$, in particular, when the ring is compressed to its gravitational radius $r_{+}, R=r_{+}$. This has been done in the nonrotating BTZ case [12] and in the rotating nonextremal BTZ case [13] where the entropic properties of the ring at the gravitational radius were deduced. In particular, it was found that the entropy of the ring is the Bekenstein-Hawking entropy given in Eq. (2), provided that the shell's temperature coincides with the Hawking temperature of the corresponding black hole. Still lacking is the study of the thermodynamics and the entropy of the extremal BTZ ring case, that might emulate the direct calculation of the entropy of an extremal BTZ black hole. For other studies related to the properties of matter systems, in particular, rotational properties in BTZ backgrounds see [14-18].

The fact that shells reflect black hole properties was found in $(3+1)$ Reissner-Nordström asymptotically flat spacetimes with an electric shell where the entropy properties of zero charge, i.e., Schwarzschild [19], nonextremal [20], and extremal [21, 22], black holes were reproduced, making these shells a very useful setting. Related to it there were the studies of the entropy for quasiblack holes $[23,24]$ and of quasistatic collapse of matter [25]. In the nonextremal case these studies found that at the gravitational radius of the shell the spacetime, and thus the corresponding black hole, has the Bekenstein-Hawking entropy of Eq. (2), where in the (3+1)-dimensional case $A_{+}=4 \pi r_{+}^{2}$. On the other hand, for extremal shells the entropy at the gravitational radius and thus the entropy of the corresponding extremal black hole, can be any wellbehaved function of the gravitational radius $r_{+}$, or since $A_{+}=4 \pi r_{+}^{2}$, the entropy can be any well-behaved function of the gravitational area $A_{+}, S=S\left(A_{+}\right)$. So among many other values, it can be zero as in Eq. (1) or $\frac{A_{+}}{4 G}$ as in Eq. (2).

The ambiguity in the entropy of extremal black holes, that can be either zero or the Bekenstein-Hawking entropy, was indeed found first in (3+1)-dimensional black holes. For extremal $(3+1)$ black holes, it was found in one approach based on the horizon topology [26] (see also [3]) that the entropy is zero $S=0$, see Eq. (1). The other approach, based on string theory calculations, yields that the entropy of extremal black holes is given by the Bekenstein-Hawking entropy, Eq. (2) [27, 28], see also [29-38]. On the other hand, for (3+1)-dimensional nonextremal black holes, the entropy is the original unambiguous Bekenstein-Hawking entropy, $S=\frac{A_{+}}{4 G}$ of Eq. (2) [39-41].

Here, we pursue further the problem of the entropy of an extremal BTZ black hole by using a shell, an extremal rotating thin shell. This is important in order to gain new insights into the entropy and other physical relevant quantities from spacetimes that possess rotation and angular momentum.

The paper is organized as follows. In Sec. II, we discuss the mechanics of an extremal rotating thin shell in $(2+1)$ dimensions with a cosmological constant. The exterior spacetime to the shell is the BTZ spacetime. In Sec. III, we study the first law of thermodynamics applied for such a thin shell, derive the thermodynamic entropy of the thin shell, and show that the entropy is a function of the gravitational area $A_{+}$only, $S=S\left(A_{+}\right)$. We also analyze the equation of state for the temperature and for the angular velocity of the shell. In Sec. IV, we consider the extremal shell with zero local temperature and take the limit to its gravitational radius, obtaining thus the properties of the corresponding extremal black hole. In Sec. V, we consider the extremal shell with some nonzero constant local temperature and take again the limit to its gravitational radius, obtaining also the properties of this extremal black hole. In Sec. VI, we discuss the nontrivial relation between mechanical and thermal, angular and linear, velocities and compare the nonextremal and extremal cases. In Sec. VII, we give some concluding remarks. In the Appendix A, we clarify the meaning of the Tolman temperature formula at a generic radius and at the shell for a spacetime with a thermal shell.

\section{THIN SHELLS IN A (2+1)-DIMENSIONAL EXTREMAL BTZ SPACETIME}

\section{A. Outer and inner spacetimes}

We consider general relativity with a cosmological constant $\Lambda$ in a $(2+1)$-dimensional spacetime. We also assume that $\Lambda<0$, so that the spacetime is asymptotically AdS, with curvature length scale $\ell=1 / \sqrt{-\Lambda}$. Throughout this paper, we work in units where the velocity of light, the Planck constant, and the Boltzmann constant are set to unity. $G$ denotes the gravitational constant in $(2+1)$ dimensions.

We introduce a timelike shell, i.e., a ring, in the $(2+1)$ dimensional spacetime, with radius $R$, which divides the 
spacetime into the outer and inner regions labelled by $(o)$ and $(i)$, respectively $[12,13]$ (see also [18]). We assume that the spacetime is vacuum everywhere off the shell. Outside the shell $(r>R)$, the spacetime is described by the extremal rotating BTZ solution, while inside the shell $(r<R)$, the spacetime is the ground state of the BTZ solution and is locally AdS. One can express the line element for the inner and outer regions by

$$
\begin{aligned}
d s_{(I)}^{2} & =-f_{(I)}(r) d t_{I}^{2}+g_{(I)}(r) d r^{2} \\
& +r^{2}\left(d \phi-\omega_{(I)}(r) d t_{(I)}\right)^{2}
\end{aligned}
$$

where $t$ is the time coordinate, $(r, \phi)$ are the radial and azimuthal coordinates, $I=o / i$ refers to the outer or inner region in relation to the shell, respectively, and the functions $f_{(I)}, g_{(I)}$ and $\omega_{(I)}$ read

$$
\begin{aligned}
& f_{(o)}(r)=\left(\frac{r}{\ell}\right)^{2}\left(1-\frac{r_{+}^{2}}{r^{2}}\right)^{2}, \quad g_{(o)}(r)=\frac{1}{f_{(o)}(r)}, \\
& \omega_{(o)}(r)=\frac{r_{+}^{2}}{\ell r^{2}}, \\
& f_{(i)}(r)=\left(\frac{r}{\ell}\right)^{2}, \quad g_{(i)}(r)=\frac{1}{f_{(i)}(r)}, \\
& \omega_{(i)}(r)=0 .
\end{aligned}
$$

The subscript $(I)$ in the time coordinate $t_{(I)}$ indicates that in general the time coordinate of the outer region $t_{(o)}$ differs from that of the inner region $t_{(i)}$. The radius $r_{+}$is the gravitational radius of the spacetime. In the extremal case, the case we consider here, the gravitational radius $r_{+}$is equal to the Cauchy radius $r_{-}, r_{+}=r_{-}$, and we stick to the usual notation $r_{+}$for such a radius. The gravitational radius $r_{+}$becomes the horizon radius if the solution is an extremal black hole or an object on the verge of becoming an extremal black hole. In the extremal case the radius $r_{+}$is given by

$$
r_{+}^{2}=4 G \ell^{2} m
$$

where $m$ is the asymptotic Arnowitt-Deser-Misner (ADM) mass. The radius $r_{+}$can also be written as $r_{+}^{2}=4 G \ell \mathcal{J}$ upon using that the spacetime angular momentum $\mathcal{J}$ and $m$ are related in the extremal case by

$$
\mathcal{J}=m \ell \text {. }
$$

We assume $m>0$. A gravitational or horizon radius $r_{+}$ corresponds to a gravitational or horizon area $A_{+}$, here a perimeter, given by

$$
A_{+}=2 \pi r_{+}
$$

The inner region $(i)$ corresponds to the ground state vacuum solution, i.e., $m=0$ and $\mathcal{J}=0$. In the junction between the outer extremal BTZ spacetime and the inner vacuum AdS spacetime, at the radius $R$, there is a stationary thin shell. We assume that the shell's character is always timelike and the shell is located outside the event horizon,

$$
R \geq r_{+}
$$

Therefore, the outer extremal region $(o)$ does not contain an event horizon $r=r_{+}$, except in the case $R=r_{+}$. One can also define the area $A$, here a perimeter, of the shell as

$$
A=2 \pi R
$$

so that Eq. (9) is written as

$$
A \geq A_{+} .
$$

An important quantity is the redshift function $k$ at some coordinate outer radius $r, k \equiv \sqrt{f_{(o)}(r)}$ which in our case is

$$
k\left(r_{+}, r\right)=\frac{r}{\ell}\left(1-\frac{r_{+}^{2}}{r^{2}}\right) .
$$

This function gets a value equal to one at the coordinate $r=r_{0}$ given by

$$
r_{0}=\frac{\ell}{2}+\sqrt{\left(\frac{\ell}{2}\right)^{2}+r_{+}^{2}},
$$

where $k\left(r_{+}, r_{0}\right)=1$. It is also of interest to display the redshift function at the position of the shell, $k \equiv \sqrt{f_{(o)}(R)}$, which in our case is

$$
k\left(r_{+}, R\right)=\frac{R}{\ell}\left(1-\frac{r_{+}^{2}}{R^{2}}\right) .
$$

This function gets a value equal to one when the shell is at the position

$$
R_{0}=\frac{\ell}{2}+\sqrt{\left(\frac{\ell}{2}\right)^{2}+r_{+}^{2}},
$$

where $k\left(r_{+}, R_{0}\right)=1$, and $R_{0}$ is always greater than both $r_{+}$and $\ell$.

\section{B. Spacetime at the junction: Properties of the extremal shell}

\section{Metric and rotation of the extremal shell}

The shell dynamics and its matter content are determined by the Israel junction conditions. The first junction condition ensures the uniqueness of the induced geometry on the shell, at $R, h_{a b}=h_{a b}^{(o)}=h_{a b}^{(i)}$, where $a, b=t, \phi$. The second junction condition determines the energy-momentum tensor of matter on the thin shell, $S_{a b}$, that compensates the jump of the extrinsic curvature tensor across the shell.

As the outer spacetime is rotating while the inner spacetime is static, in order to match these two regions, the shell at $r=R$ must corotate with the outer BTZ region. We introduce a coordinate system corotating with the shell by adopting a new angular coordinate $d \psi$ such that

$$
d \psi=d \phi-\omega_{(I)}(R) d t_{(I)}
$$


The line element given in Eq. (3) is then written as

$$
\begin{aligned}
d s_{(I)}^{2} & =-f_{(I)}(r) d t_{(I)}^{2}+g_{(I)}(r) d r^{2} \\
& +r^{2}\left(d \psi-o_{(I)}(r) d t_{(I)}\right)^{2},
\end{aligned}
$$

where we have introduced

$$
o_{(I)}(r)=\omega_{(I)}(r)-\omega_{(I)}(R),
$$

so that at the position of the shell $o_{(I)}(R)=0$ and the line element is diagonal. Also, from Eqs. (4) and (5),

$$
\omega_{(o)}(R)=\frac{r_{+}^{2}}{\ell R^{2}},
$$

and

$$
\omega_{(i)}(R)=0,
$$

respectively. The induced line element on the shell at $R$ uniquely determined by the first junction condition is given by

$$
d s_{R}^{2}=-d \tau^{2}+R^{2} d \psi^{2}, \quad R=R(\tau) .
$$

The proper time on the shell $\tau$ is defined by

$$
\begin{aligned}
d \tau & =\sqrt{f_{(o)}(R) d t_{(o)}^{2}-g_{(o)}(R) d R^{2}} \\
& =\sqrt{f_{(i)}(R) d t_{(i)}^{2}-g_{(i)}(R) d R^{2}} .
\end{aligned}
$$

Since we are interested in a quasistatic process we always assume that $\frac{d R}{d \tau}=0$ and $\frac{d^{2} R}{d \tau^{2}}=0$.

\section{Energy-momentum tensor of the extremal shell}

The rotating thin shell is supported by an imperfect fluid with an energy-momentum tensor $S^{a}{ }_{b}$, such that the nonzero components are $S_{\tau}^{\tau}=-\sigma, S_{\psi}^{\psi}=p$, and $S^{\tau}{ }_{\psi}=j$, where $\sigma, p$, and $j$, represent the energy density of the shell, the pressure in the shell, and the angular momentum flux density of the shell, respectively. The second junction condition gives

$$
\begin{aligned}
\sigma & =\frac{r_{+}^{2}}{8 \pi G \ell R^{2}}, \\
j & =\frac{r_{+}^{2}}{8 \pi G \ell R}, \\
p & =\frac{r_{+}^{2}}{8 \pi G \ell R^{2}} .
\end{aligned}
$$

Thus, $\sigma=p=j / R$. The extremal rotating shell obeys both the weak and dominant energy conditions [18].

Defining the local proper mass and angular momentum of the shell by $M=2 \pi R \sigma$ and $J=2 \pi R j$, respectively, and using Eqs. (23) and (24), we obtain

$$
\begin{aligned}
M & =2 \pi R \sigma=\frac{r_{+}^{2}}{4 G \ell R}, \\
J & =2 \pi R j=\frac{r_{+}^{2}}{4 G \ell} .
\end{aligned}
$$

3. Relation between global extremal spacetime quantities and local extremal shell quantities

The spacetime quantities $m$ and $\mathcal{J}$ are related to the shell quantities $M$ and $J$. From Eqs. (6) and (26), one finds that the local proper mass of the shell $M$ is related to the ADM mass $m$ by

$$
m=\frac{M R}{\ell}
$$

From Eq. (27), one sees that the angular momentum of the shell $J$ is independent of the position of the shell $R$, and from Eqs. (6) and(7), we see that it is identical to the angular momentum of the outer BTZ spacetime,

$$
\mathcal{J}=J
$$

We would like to emphasize that in our case the inner region is a $(2+1)$-dimensional spacetime locally AdS and it has zero ADM mass and zero angular momentum. In the more complex case that the region inside the shell contains instead a BTZ black hole, then the total ADM mass and angular momentum of the outer spacetime defined at infinity would include in addition the ADM mass and angular momentum of the interior black hole.

\section{FIRST LAW OF THERMODYNAMICS, ENTROPY OF AN EXTREMAL ROTATING THIN SHELL IN A BTZ SPACETIME, AND THE EQUATIONS OF STATE}

\section{A. First law of thermodynamics of an extremal rotating thin shell in a BTZ spacetime}

Now, we turn to the thermodynamics of the thin shell. Following [13], we assume that the rotating shell has a thermodynamic angular velocity $\Omega$ and is in thermal equilibrium, with local temperature $T$ and entropy $S$. The entropy $S$ of a system can be expressed as a function of the state independent variables. One can take as state independent thermodynamic variables for the thin shell the proper mass $M$, the area of the shell $A$, and the angular momentum of the shell $J$. Thus, the entropy of the shell is a function of these quantities through the first law of thermodynamics which reads

$$
T d S=d M+p d A-\Omega d J .
$$

To obtain the entropy $S$, in general we have to specify the three equations of state $T(M, A, J), p(M, A, J)$, and $\Omega(M, A, J)$, namely, the temperature, pressure, and angular velocity equations of state, respectively. To proceed in this direction, define the inverse local temperature of the shell as

$$
\beta=\frac{1}{T} .
$$


Note further that in a $(2+1)$-dimensional spacetime the area $A$ of the shell, actually a perimeter in common usage, is given by Eq. (10) so that it is mathematically equivalent to the position $R$ except for the trivial factor $2 \pi$, i.e., we can make use of the variable $R$ instead of $A$. Using Eq. (31), the first law of thermodynamics (30) now reads in the $(M, R, J)$ variables

$$
d S=\beta d M+2 \pi \beta p d R-\beta \Omega d J .
$$

We need to give the equations of state for $\beta(M, R, J)$, $p(M, R, J)$, and $\Omega(M, R, J)$.

\section{B. Entropy of an extremal rotating thin shell in a BTZ spacetime}

We can make progress using first, for the time being, the equation of state for the pressure $p$. Through the junction condition, i.e., through the spacetime mechanics, $p$ is indeed fixed by Eq. (25). Changing to the variables $M$ and $R$, and using Eqs. (25) and (26), valid for an extremal shell, one finds that the equation of state for the pressure $p$ can be written as

$$
p(M, R)=\frac{M}{2 \pi R} .
$$

Also, one can still take advantage of Eqs. (26) and (27). Through these equations, we obtain

$$
J=M R .
$$

Equation (34) gives that $J, M$, and $R$ are not independent, with $d J=R d M+M d R$. Putting Eqs. (33) and (34) into the first law Eq. (32), we obtain the differential of entropy $d S=\frac{\beta}{R}(1-\Omega R) d(M R)$. It is then useful to define the the thermodynamic rotational velocity of the shell $V$ by

$$
V=\Omega R, \quad 0 \leq V \leq 1,
$$

where the constraint $V \leq 1$ ensures that the maximum velocity is the velocity of light. Then, the entropy of the extremal rotating shell obeys

$$
d S=\frac{\beta}{R}(1-V) d(M R) .
$$

Clearly, from Eq. (26), i.e., $M R=\frac{r_{+}^{2}}{4 G \ell}$, it is natural to pass from the variables $M R$ to the variable $r_{+}$. In this variable $r_{+}$, the differential of entropy Eq. (36) can further be reduced to

$$
d S=\frac{r_{+}}{2 G \ell} \frac{\beta}{R}(1-V) d r_{+} .
$$

It is manifest that Eq. (37) has to be written as

$$
d S=s\left(r_{+}\right) d r_{+},
$$

where

$$
s\left(r_{+}\right)=\frac{r_{+}}{2 G \ell} \frac{\beta}{R}(1-V),
$$

is actually the integrability condition for Eq. (37) and where $\beta$ and $V$ are arbitrary functions of $\left(r_{+}, R\right)$, but $s\left(r_{+}\right)$is an arbitrary function of $r_{+}$alone. Thus, the relation (38) indicates that the entropy $S$ of the shell is a function of the gravitational radius of the shell $r_{+}$only,

$$
S=S\left(r_{+}\right), \quad R>r_{+},
$$

where we have set a constant of integration to zero. Since one can trade $r_{+}$for $A_{+}$trivially through Eq. (8), we write Eq. (40) in the more visual form

$$
S=S\left(A_{+}\right), \quad A>A_{+} .
$$

Depending on the choice of $s\left(r_{+}\right)$in Eq. (39), the entropy $S\left(r_{+}\right)$, or equivalently $S\left(A_{+}\right)$, of the rotating shell in the BTZ spacetime can take a wide range of values.

Now, in the variables $\left(r_{+}, R\right)$ (or if one prefers, $\left.\left(A_{+}, A\right)\right)$, which are the natural variables for calculation in this problem, one has two remaining functions of state, $\beta\left(r_{+}, R\right)$ and $V\left(r_{+}, R\right)$. These two functions of state, $\beta\left(r_{+}, R\right)$ and $V\left(r_{+}, R\right)$, are arbitrary as long as they obey the thermodynamic constraint (39). The extremal rotating shell is in this sense quite special.

\section{Equations of state for the inverse temperature $\beta$ and for the rotational velocity $V$}

\section{Inverse temperature $\beta$ equation of state}

Now, we prepare a given extremal shell at a generic radius $R$. The unique integrability condition (39) is quite general and does not impose per se a restriction on the temperature distribution. However, thermodynamic systems have to obey the Tolman formula for the temperature or, equivalently, for the inverse local temperature.

The Tolman temperature formula, by its very meaning, states that the coordinate dependence of the temperature obeys some restriction. The Tolman temperature formula for spherical systems of the type we are using here is $T(r)=T_{0} / k(r)$ where the dependence on $r$, the local radial coordinate, exists only in the redshift factor $k$, see Appendix A for a thorough discussion on the Tolman formula for shells. The quantity $T_{0}$ may depend on the parameters of the system. In our case, the gravitational radius $r_{+}$and the radius of the shell $R$ are examples of such parameters, such that $T_{0}=T_{0}\left(r_{+}, R\right)$. For a given shell's position, fixed $R$, we can consider the Tolman formula in the whole outer space. For some other $R$, the configuration changes, and for that new configuration we can consider again the Tolman formula in the new whole outer space. More precisely, suppose that we have a shell at position $R$ with intrinsic gravitational radius $r_{+}$at some given temperature. The local temperature of the spacetime at some specific radius $r$ is $T$, say. 
The Tolman formula relates the local temperature $T$ to a constant temperature parameter $T_{0}$. In the case under discussion, $T_{0}$ has the meaning of the local temperature at $r=r_{0}$, where $k\left(r_{0}, r_{+}\right)=1$ (see Eq. (13)). In brief, the Tolman formula states that $T$ at $r, T(r)$, is a function of a temperature $T_{0}$, at the radius $r_{0}$ where $k\left(r_{0}, r_{+}\right)=1$. $T_{0}$ is itself a function of the characteristics of the system, $r_{+}$and $R$ in our case,

$$
T_{0}=T_{0}\left(r_{+}, R\right) .
$$

Thus, dividing by the redshift function at $r$ given in Eq. (12), the Tolman temperature formula in full is

$$
T\left(r_{+}, R, r\right)=\frac{T_{0}\left(r_{+}, R\right)}{k\left(r_{+}, r\right)} .
$$

Inverting this equation, and using Eq. (31), yields the required Tolman formula for the inverse temperature $\beta$, i.e.,

$$
\beta\left(r_{+}, R, r\right)=b\left(r_{+}, R\right) k\left(r_{+}, r\right),
$$

where

$$
b\left(r_{+}, R\right)=\frac{1}{T_{0}\left(r_{+}, R\right)} .
$$

In this way, one interprets $b\left(r_{+}, R\right)$ as the inverse temperature at the radius $r=r_{0}$ for which $k=1$. Conversely, $\beta$ is the inverse temperature at $r$, blueshifted or redshifted with factor $k$ from the inverse temperature at the position where $k=1$.

Now, on the shell, $r=R$, so the Tolman formula there is $\beta\left(r_{+}, R, r=R\right)=b\left(r_{+}, R\right) k\left(r_{+}, r=R\right)$, or simplifying the notation,

$$
\beta\left(r_{+}, R\right)=b\left(r_{+}, R\right) k\left(r_{+}, R\right) .
$$

If the shell happens to be at $R=R_{0}$, where $R_{0}=\frac{\ell}{2}+$ $\sqrt{\left(\frac{\ell}{2}\right)^{2}+r_{+}^{2}}$, then $k\left(r_{+}, R_{0}\right)=1$, see Eq. (15), and so there $\beta\left(r_{+}, R_{0}\right)=b\left(r_{+}, R_{0}\right)$.

It is important to note that the formula (46) for ab initio extremal shells is more comprehensive, and so different, than the one found for extremal shells formed from taking the limit of nonextremal shells [13]. In [13] it was found that for nonextremal shells, with radius $R$ and gravitational and Cauchy radii $r_{+}$and $r_{-}$, the following Tolman equation at the shell's radius $R$, found from the integrability conditions, holds, i.e., $\beta\left(r_{+}, r_{-}, R\right)=b\left(r_{+}, r_{-}\right) k\left(r_{+}, r_{-}, R\right)$, where $k\left(r_{+}, r_{-}, R\right)=\frac{R}{\ell} \sqrt{\left(1-\frac{r_{+}^{2}}{R^{2}}\right)\left(1-\frac{r_{-}^{2}}{R^{2}}\right)}$ is the redshift function in the nonextremal case. Taking then, from the nonextremal shell, the extremal shell limit $r_{+} \rightarrow r_{-}$ and noting that $k\left(r_{+}, r_{-}, R\right)=k\left(r_{+}, R\right)$ in this limit where $k=k\left(r_{+}, R\right)$ is given in Eq. (14), one finds $\beta\left(r_{+}, R\right)=b\left(r_{+}\right) k\left(r_{+}, R\right)[13]$. Note the difference: the limit of a nonextremal shell to an extremal shell gives $b=b\left(r_{+}\right)$, and $b$ is a function of $r_{+}$alone [13]. On the other hand, when one has an extremal shell ab initio, one finds $b=b\left(r_{+}, R\right)$, see Eq. (46), i.e., in this more comprehensive case, $b$ is a function not only of $r_{+}$but also of $R$.

The difference comes of course from the different integrability conditions arising in the nonextremal and extremal cases. For an ab initio extremal shell, the only integrability condition (39) is too general and gives $b=b\left(r_{+}, R\right)$ as in Eq. (46). For a nonextremal shell, the three integrabilty conditions are very restrictive, and when one takes the extremal limit, the memory of this restrictiveness remains, so $b=b\left(r_{+}\right)$.

\section{Rotational velocity $V$ equation of state}

With the choice for the inverse temperature equation of state (46), we find from Eq. (39) that the rotational velocity of the shell has the functional form

$$
V\left(r_{+}, R\right)=\frac{R}{\ell k\left(r_{+}, R\right)}\left(g\left(r_{+}, R\right)-\frac{r_{+}^{2}}{R^{2}}\right),
$$

where we have defined

$$
g\left(r_{+}, R\right)=1-\frac{2 G \ell^{2}}{r_{+} b\left(r_{+}, R\right)} s\left(r_{+}\right) .
$$

For an ab initio extremal shell, $g$ has a dependence on $r_{+}$ as well as on $R$. For a nonextremal shell and taking the limit to the extremal shell [13] the corresponding function depends only on $r_{+}$. As in the equation of state for the inverse temperature, this comes from the very different integrability conditions in each case. Note that $g$ in Eq. (48) corresponds to $c$ in Eq. (59) of [13] with $g=c r_{+}^{2}$, but now here $g$ in general has the dependence on $R$ as well as $r_{+}$due to the different integrability conditions as discussed.

With the definition of $g$ in Eq. (48), we have from Eq. (39) the useful formula

$$
s\left(r_{+}\right)=\frac{r_{+}}{2 G \ell^{2}} b\left(r_{+}, R\right)\left(1-g\left(r_{+}, R\right)\right),
$$

which shows that, although $b$ and $g$ are functions of $r_{+}$ and $R$, their combination is a function of $r_{+}$alone. Also, from the definition of $g$ in Eq. (48), we have another useful formula,

$$
V\left(r_{+}, R\right)=1-\frac{R}{\ell k\left(r_{+}, R\right)}\left(1-g\left(r_{+}, R\right)\right) .
$$

We see that the velocity $V \rightarrow 1$ when $g \rightarrow 1$, i.e., when $b \rightarrow \infty$ according to Eq. (48), or $T_{0} \rightarrow 0$. In this respect, there is a direct remarkable interesting relation between the unattainability of the absolute zero of temperature and the impossibility for a material body to reach the velocity of light. 


\section{Explicit computation of the entropy of the shell}

For the explicit computation of the entropy $S$ of the shell, see Eq. (40) (or Eq. (41)), we have to specify the equations $b\left(r_{+}, R\right)$ and $g\left(r_{+}, R\right)$ which determine the thermodynamic properties of the shell. In this paper, we do not proceed in this way but, instead, focus on Eqs. (46) and (47) and study the particular cases for which we can take the limit to the extremal black hole, $R \rightarrow r_{+}$.

\section{ENTROPY IN THE EXTREMAL BTZ BLACK HOLE LIMIT: EXTREMAL THIN SHELL WITH ZERO LOCAL TEMPERATURE $T$ AT THE GRAVITATIONAL RADIUS}

We will now study the extremal black hole limit in the sense that we take quasistatically the extremal shell to its own gravitational radius, $R=r_{+}$.

In this procedure of going quasistatically to the gravitational radius $R=r_{+}$, we have to prepare in advance the shell. In first place, we put the shell at some radius $R>r_{+}$and in addition choose the functions $\beta$ and $V$, or $b$ and $g$, in an appropriate manner. After doing this, we take the shell to $R=r_{+}$. In second place, we stipulate $b$ and so $\beta$. We know that the Hawking temperature $T_{H}$ for a BTZ black hole is measured at $r_{0}$, i.e., $T_{0}=T_{H}$, see e.g., [6]. For an extremal black hole, this temperature is zero $T_{H}=0$. We assume that the equality $T_{0}=T_{H}$ is valid for our shell since otherwise the backreaction of quantum fields would destroy it when the shell approaches the horizon. Now, the temperature $T_{0}$ is precisely related to our $b, b\left(r_{+}, R\right)=1 / T_{0}\left(r_{+}, R\right)$, see Eq. (45). But since $T_{0}=T_{H}=0$, we have, for a shell at radius $R$, to set $b=\infty$. Thus, from Eq. (44), $\beta\left(r_{+}, R, r\right)=\infty$ and in particular $\beta\left(r_{+}, R, r=R\right) \equiv \beta\left(r_{+}, R\right)=\infty$. The temperature at the shell is zero. We can now change the radius of the shell quasistatically, and the same rationale applies, since we always want $T_{0}=T_{H}=0$, i.e., $b=\infty$. In third place, we find $g$ and $V$. From Eq. (49), we find that when $b=\infty$ then $g=1$. Let us suppose that we start with a configuration in which $g$ is not equal to 1 exactly and $b$ is large but finite. Then, $1-g=s\left(2 G \ell^{2} / r_{+}\right) / b$, for some well-specified $s$. We are interested in the limit in which $b \rightarrow \infty, g \rightarrow 1, V \rightarrow 1$. To trace in more detail this limit, we can choose $g$ as close to 1 as we want and $T_{0}$ as small as we like, i.e., $b$ as large as we like. In the end, keeping the product fixed in Eq. (49) (for a given $\left.r_{+}\right)$, we can take the limit of $g$ to 1 and $T_{0}$ to zero, i.e., $b$ to infinity. We see that the shell at $R>r_{+}$has been prepared with $T_{0}=0$, i.e., $b=\infty$, and $g=1$, such that $\frac{r_{+}}{2 G \ell^{2}} b(1-g)=s$ and so $\frac{r_{+}}{2 G \ell} \frac{\beta}{R}(1-V)=s$, with thus $\beta=\infty$ and $V=1$. Since $V=1$, the shell rotates with the velocity of light precisely in this limit. The shell is now correctly prepared. Having made the correct preparations on the shell, and as long as $R \geq r_{+}$and imposing that Eq. (49) is always obeyed for some fixed $s\left(r_{+}\right)$, we can send it to its gravitational radius $r_{+}$.
Let us then send the extremal shell to its own gravitational radius $R=r_{+}$. In doing so we are taking the extremal black hole limit. Since the entropy differential for the shell depends only on $r_{+}$through the function $s\left(r_{+}\right)$that is arbitrary, see Eq. (39), we see that the entropy of the extremal shell in the extremal black hole limit is given by

$$
S=S\left(r_{+}\right), \quad R=r_{+},
$$

or, in terms of the horizon area if one prefers,

$$
S=S\left(A_{+}\right), \quad A=A_{+} .
$$

This is the extremal black hole limit of an extremal shell. This type of configuration, a matter system at its own gravitational radius, is called a quasiblack hole [23, 24]. Thus the entropy of the extremal black hole can be any well-behaved function of $r_{+}$, or $A_{+}$, which depends on the constitution of matter that collapses to form the extremal BTZ black hole. Depending on the choice of $s\left(r_{+}\right)$, in turn of $\beta$ and $V$, we can obtain any function of $r_{+}$, or $A_{+}$, for the entropy $S$ of the extremal black hole. This result is quite different from the nonextremal case discussed in [13], where the entropy of the shell for which the temperature coincides with the Hawking temperature can only take the form of the Bekenstein-Hawking entropy $S\left(A_{+}\right)=\frac{A_{+}}{4 G}$.

Our preceding calculations and discussion were exact. Now, we can speculate on ways to constrain the entropy function $S\left(A_{+}\right)$for the extremal black hole. For the nonextremal black holes, the entropy is $S\left(A_{+}\right)=\frac{A_{+}}{4 G}$. This expression is found when one takes the shell to its own gravitational radius and assumes that the shell takes the Hawking temperature. In this case, the pressure at the shell blows up, $p \rightarrow \infty[12,13]$. This blowing up of the pressure can be interpreted as the excitation of all possible degrees of freedom and the corresponding black hole takes the Bekenstein-Hawking entropy, the maximum possible entropy. Taking the extremal limit from a nonextremal black hole, one finds that in this particular limit the extremal black hole entropy is the BekensteinHawking entropy [12, 13] (see also [23, 24]). Thus, this suggests that the maximum entropy that an extremal black hole can take is the Bekenstein-Hawking entropy. Therefore, in this sense, the range of values for the entropy of an extremal black hole is

$$
0 \leq S\left(r_{+}\right) \leq \frac{\pi r_{+}}{2 G}
$$

or, in terms of $A_{+}$,

$$
0 \leq S\left(A_{+}\right) \leq \frac{A_{+}}{4 G} .
$$

The result (51), or equivalently (52), is a quite similar result to the case of the extremal charged shell in a $(3+1)$ Reissner-Nordström spacetime [21, 22]. As for the extremal electrically charged shells [21, 22], we then 
constrained the entropy function $S\left(r_{+}\right)$, or $S\left(A_{+}\right)$, for the extremal black hole. For the nonextremal ReissnerNordström black holes, the entropy is given by the Bekenstein-Hawking formula. In this case, when the shell is taken to its own gravitational radius, the pressure at the shell diverges, $p \rightarrow \infty$ as $k^{-1}$ (see (54) of [13]), and the spacetime is assumed to take the Hawking temperature. Thus all possible degrees of freedom on the shell are excited and the black hole formed as the limit of the shell to its gravitational radius takes the Bekenstein-
Hawking entropy $S\left(r_{+}\right)=\frac{A_{+}}{4 G}$ as the maximal entropy. This suggests that the range of values for the entropy of the extremal black hole in the $(3+1)$ dimensions is given by Eq. (54) [21], as in the case of the rotating black hole in the $(2+1)$ dimensions considered here.

In Table I, we summarize the thermodynamic properties of the extremal thin shell at its own gravitational radius with zero local limiting temperature.

\begin{tabular}{|cc|cc|c|c|c|}
\hline$T_{0}$ & $b$ & $T$ & $\beta$ & Backreaction & $V$ & Entropy \\
\hline 0 & $\infty$ & 0 & $\infty$ & Finite & 1 & $0 \leq S\left(A_{+}\right) \leq \frac{A_{+}}{4 G}$ \\
\hline
\end{tabular}

TABLE I: The extremal shell with zero local temperature at its own gravitational radius.

So, we have found through a thin shell approach that the $(2+1)$-dimensional extremal rotating BTZ black hole has an entropy $S=S\left(A_{+}\right)$as we had found for the $(3+1)$-dimensional extremal Reissner-Nordström electrically charge black hole [21, 22], and again suggested $0 \leq S\left(A_{+}\right) \leq \frac{A_{+}}{4 G}$, see Eq. (54). The extremal black hole entropy was discussed originally in a $(3+1)$-dimensional black hole context. It was found in [26] that the entropy of an extremal $(3+1)$-dimensional black hole is zero, $S=0$, Eq. (1). This proposal was substantiated by topology arguments. The $(2+1)$-dimensional metric does not contain one of the angle coordinates when compared to the $(3+1)$-dimensional metric, but this is unessential since the main arguments concern the topology of the $(\tau, r)$ submanifold, where $\tau \equiv i t$ is the Euclidean time, see also [3] for $(2+1)$-dimensional BTZ black hole. This reasoning is purely classical, and inclusion of the backreaction due to quantum fields can destroy this picture. On the other hand, the proposal put forward by string theory leads to $S=\frac{A_{+}}{4 G}$, Eq. (2), i.e., to a Bekenstein-Hawking entropy for extremal black hole [27, 28], see [2] for the BTZ black hole. Our conclusion that $0 \leq S\left(A_{+}\right) \leq \frac{A_{+}}{4 G}$, see Eq. (54), incorporates both the $S=0$ and the $S=\frac{A_{+}}{4 G}$ results.

\section{ENTROPY IN THE EXTREMAL BTZ} BLACK HOLE LIMIT: EXTREMAL THIN SHELL WITH NONZERO LOCAL TEMPERATURE $T$ AT THE GRAVITATIONAL RADIUS

Now, we take again the extremal black hole limit but assume that the extremal shell has another equation of state with a nonzero local temperature. Specifically, at any $r>r_{+}$, we consider the following temperature equation of state,

$$
T_{0}\left(r_{+}, R\right)=\bar{T}_{0}\left(r_{+}\right) k\left(r_{+}, R\right),
$$

where $\bar{T}_{0}$ is independent of $R$. Then, from Eq. (43), the temperature $T$ is $T\left(r_{+}, R, r\right)=\bar{T}_{0}\left(r_{+}\right) \frac{k\left(r_{+}, R\right)}{k\left(r_{+}, r\right)}$. At the shell $r=R$, one gets

$$
T\left(r_{+}, R\right)=\bar{T}_{0}\left(r_{+}\right) .
$$

In terms of the inverse temperatures Eq. (55) translates into

$$
b\left(r_{+}, R\right)=\frac{\bar{b}\left(r_{+}\right)}{k\left(r_{+}, R\right)},
$$

where

$$
\bar{b}\left(r_{+}\right) \equiv \frac{1}{\bar{T}_{0}\left(r_{+}\right)},
$$

is independent of $R$. Then, from Eq. (44), the local inverse temperature $\beta$ at $r$ is $\beta\left(r_{+}, R, r\right)=\bar{b}\left(r_{+}\right) \frac{k\left(r_{+}, r\right)}{k\left(r_{+}, R\right)}$. Likewise, at the shell, $r=R$, one gets

$$
\beta\left(r_{+}, R\right)=\bar{b}\left(r_{+}\right)
$$

is constant and finite.

Combining (48) with (57), we find that the rotational velocity equation of state can be written as

$$
1-g\left(r_{+}, R\right)=k\left(r_{+}, R\right)\left(1-\bar{g}\left(r_{+}\right)\right),
$$

where we have defined

$$
\bar{g}\left(r_{+}\right) \equiv 1-\frac{2 G \ell^{2}}{r_{+} \bar{b}\left(r_{+}\right)} s\left(r_{+}\right),
$$

which is independent of $R$ and is assumed to be in the range $0<\bar{g}\left(r_{+}\right)<1$.

We now take the limit to the gravitational radius of the shell, $R \rightarrow r_{+}$. In this limit, $k \rightarrow 0$. So, supposing $\bar{T}_{0}$ finite, which we do, we see from Eq. (55) that $T_{0}\left(r_{+}, R\right)=0$. Since $\bar{T}_{0}$ is finite, the local temperature 
$T$ at the shell is nonzero but finite, since $T=\bar{T}_{0}$ from Eq. (56), and hence the quantum backreaction remains finite even when the shell is taken to its gravitational radius $R=r_{+}$. Since $T$ is finite $\beta$ given in Eq. (59) is also finite, and from Eq. (39), we find that the shell with nonzero local temperature rotates with thermal velocity less than the velocity of light $V<1$. Finally, the entropy of the shell obtained by integrating Eq. (38) is well behaved and can take any function of $r_{+}$, or $A_{+}$. Thus, using the same arguments as before, we can write that the entropy of this extremal shell in the extremal BTZ black hole limit also obeys

$$
0 \leq S\left(A_{+}\right) \leq \frac{A_{+}}{4 G}
$$

In Table II, we summarize the thermodynamic properties of the extremal thin shell at its own gravitational radius with a local temperature $T=\bar{T}_{0}$.

\begin{tabular}{|c|c|c|c|c|}
\hline $\begin{array}{|ll|}T_{0} & b \\
\end{array}$ & $\begin{array}{ll}T & \beta \\
\end{array}$ & Backreaction & \begin{tabular}{|l}
$V$ \\
\end{tabular} & Entropy \\
\hline 0 & Nonzero and Finite & finite & $<1$ & $0 \leq S$ \\
\hline
\end{tabular}

TABLE II: The extremal shell with nonzero local temperature at its own gravitational radius.

\section{DISCUSSION ON THE ANGULAR, AND THE CORRESPONDING LINEAR, VELOCITIES OF ROTATING THIN SHELLS}

\section{A. Mechanical angular velocities}

It is instructive to rewrite the formula for the line element outside of the shell (see Eqs. (3) and (4)) as

$d s_{(o)}^{2}=-f_{(o)}(r) d t_{(o)}^{2}+g_{(o)}(r) d r^{2}+r^{2}\left(d \phi-\omega_{(o)}(r) d t_{(o)}\right)^{2}$.

Now, define

$$
\omega_{R} \equiv \omega_{(o)}(R)
$$

Static observers sitting at infinity, $r=\infty$, have an AdS metric, since the BTZ metric turns into an asymptotically AdS metric at infinity. These observers do not rotate relative to this AdS spacetime. Thus, observers sitting at infinity see a rotation of the shell with $\omega_{R}$. Here we keep the discussion quite general, for the extremal BTZ case $\omega_{R}$ is given in Eq. (19), i.e., $\omega_{R}=\frac{r_{+}^{2}}{\ell R^{2}}$.

At the shell $r=R$, the metric (63) becomes

$$
d s_{R}^{2}=-d \tau^{2}+R^{2} d \psi^{2}, \quad R=R(\tau),
$$

where $\tau$ is the proper time at the shell and in terms of $d t_{(o)}$ is $d \tau=\sqrt{f_{(o)}(R)} d t_{(o)}=k\left(r_{+}, R\right) d t_{(o)}$ (see also (14)), and we have chosen a new angular coordinate $\psi$,

$$
\psi=\phi-\omega_{R} t_{(o)},
$$

such that the metric is displayed as diagonal (see also Eq. (16)). The angular velocity $\omega_{R}$ defined in Eq. (64), appears thus quite naturally in Eq. (66) and is one of a number of interesting mechanical angular velocities in this problem. Let us display the others, that we name $\bar{\omega}$, $\omega$, and $\omega_{\infty}$.
From Eqs. (65) and (66) one deduces that an observer comoving with the shell has $\psi=$ constant. Another observer on the shell moving with respect to this comoving observer has angular velocity $\bar{\omega}$ with respect to the proper time on the shell $\tau$ given by

$$
\bar{\omega}=\frac{d \psi}{d \tau} .
$$

This same observer has an angular velocity $\omega$ with respect to $t_{(o)}$ given by $\omega=\frac{d \psi}{d t_{(o)}}=\frac{d \tau}{d t_{(o)}} \frac{d \psi}{d \tau}=k \bar{\omega}$, where here $k \equiv k\left(r_{+}, R\right)$ to simplify the notation, i.e.,

$$
\omega=\frac{d \psi}{d t_{(o)}}=k \bar{\omega} .
$$

Now, from Eq. (63), the coordinate $\phi$ is the angular coordinate defined at infinity. Define then the angular velocity $\omega_{\infty}$ of an observer on the shell as seen by the coordinate $\phi$ and in terms of $t_{(o)}$ as

$$
\omega_{\infty}=\frac{d \phi}{d t_{(o)}} .
$$

We can now give a relation between $\omega, \omega_{\infty}$, and $\omega_{R}$, or between $\bar{\omega}, \omega_{\infty}, \omega_{R}$, and $k$. Clearly, from Eq. (66),

$$
\frac{d \psi}{d t_{(o)}}=\frac{d \phi}{d t_{(o)}}-\omega_{R},
$$

so that, using Eqs. (68) and (69) in (70), we find

$$
\omega=\omega_{\infty}-\omega_{R}
$$

If we prefer to use $\bar{\omega}$, i.e., to use the proper time coordinate $\tau$, then from the help of Eqs. (67) and (68) we find

$$
\bar{\omega}=\frac{\omega_{\infty}-\omega_{R}}{k} .
$$


Expression (72) is quite general. One can specialize. For instance, the special choice of $\bar{\omega}$ for which a shell's observer detects no angular momentum flux density was given by Eq. (39) of [18], i.e., $\bar{\omega}=\frac{r_{-}}{r_{+} R} \sqrt{\frac{R^{2}-r_{+}^{2}}{R^{2}-r_{-}^{2}}}$. Note that $\omega_{R}$ is annotated as $\Omega$ in [18], the angular velocity of the shell with respect to infinity. We use here the notation $\Omega$ for a quite different quantity, the thermodynamic angular velocity on the shell, see Eq. (30). The linear velocities corresponding to $\bar{\omega}, \omega$, and $\omega_{\infty}$ are $\bar{v}=\bar{\omega} R$, $v=\omega R$, and $v_{\infty}=\omega_{\infty} R$. Angular and linear velocities share the same properties.

It is also instructive and important to point out the analogy with the black hole case. In this context, Eq. (72) is similar to the expression for the angular velocity of the heat bath surrounding a $(2+1)$-dimensional rotating black hole,

$$
\omega_{\mathrm{hb}}=\frac{\omega_{\mathrm{bh}}-\omega_{\mathrm{zamo}}}{k},
$$

where $\omega_{\mathrm{hb}}$ is the heat bath angular velocity, $\omega_{\mathrm{bh}}$ is the black hole angular velocity, $\omega_{\text {zamo }}$ is the angular velocity of a zero angular momentum observer (ZAMO), and $k$ in this formula is the redshift function at the ZAMO radius, see Eq. (13) of [5]. So, at a first glance, one may identify $\bar{\omega} \equiv \omega_{\mathrm{hb}}, \omega_{\infty} \equiv \omega_{\mathrm{bh}}$, and $\omega_{R} \equiv \omega_{\text {zamo }}$. There is, however, an important difference between Eqs. (72) and (73). For a black hole, the quantity $\omega_{\text {hb }}$ enters both the mechanical and thermodynamic relations (see [5] for details), so the mechanical and thermodynamic angular velocities coincide. However, for a shell the relationship between both angular velocities is much more subtle as we now discuss.

\section{B. Mechanical and thermodynamic angular and linear velocities for a rotating shell: The nonextremal and extremal cases}

\section{The problem}

Consideration of thermal shells in $(2+1)$ dimensions in the present paper as well in previous ones [18] (see also [13]) revealed interesting subtleties otherwise hidden. At first sight, the shell thermodynamic angular velocity $\Omega$ that appears in the first law of thermodynamics, Eq. (30), should be immediately identified with the shell mechanical angular velocity of a rotating shell fluid $\bar{\omega}$, Eq. (72), in the same way as the quantity $\omega_{\mathrm{hb}}$ is both the mechanical and thermodynamic angular velocity in the black hole case, as discussed above (see [5]).

However, the two angular velocities, namely, the thermodyamic angular velocity $\Omega$ and the mechanical velocity $\bar{\omega}$, are indeed conceptually different, they have different physical meanings. The quantity $\Omega$ is the quantity ascribed to a thermodynamic system as a whole, it is calculated from a pure thermodynamic approach [13].
On the other hand, the quantity $\bar{\omega}$ represents the angular velocity of the effective perfect fluid that fills the shell, i.e., it is obtained from geometry and mechanics, namely, by gluing two metrics, the BTZ metric and the zero mass BTZ metric on the different sides of the shell, calculating the corresponding energy-momentum tensor and determining the angular velocity of the effective fluid in terms of which the vanishing angular momentum flux is observed [18]. The same rationale applies of course for the corresponding linear velocities, $V=\Omega R$ and $\bar{v}=\bar{\omega} R$, i.e., they are conceptually different.

So, a priori, it is not obvious at all, whether or not, and under what conditions, these two velocities can be identified. It is thus instructive to compare this issue for extremal and nonextremal shells.

\section{Extremal shell}

For an extremal shell the situation is interesting and different from what one would expect.

Indeed, the two velocities $\Omega$ and $\bar{\omega}$, or $V$ and $\bar{v}$, do not need to coincide at all, as the integrability condition (39) does not restrict the form of the function $\Omega$ in the extremal case. We will explain this fact now.

The thermodynamic angular velocity $\Omega$ presents new features. As it is argued above in Sec. IV, when $T=0$, then we must select

$$
\Omega=\frac{1}{R}, \quad T=\frac{1}{\beta}=0,
$$

so that,

$$
V=1,
$$

in the extremal limit under discussion, to ensure the finiteness of $s\left(r_{+}\right)$. This is the extremal black hole limit from an extremal thin shell with zero local temperature $T$, see also Table I. However, when $T \neq 0$, see Sec. V, any thermodynamic angular velocity $\Omega$ obeying

$$
\Omega<\frac{1}{R}, \quad T=\frac{1}{\beta} \neq 0,
$$

i.e.,

$$
V<1,
$$

is suitable provided that $T=\beta^{-1}$ remains nonzero in the extremal black hole limit $R \rightarrow r_{+}$, see Eq. (39). This is the extremal black hole limit from an extremal thin shell with nonzero local temperature $T$, see also Table II.

The mechanical angular velocity $\bar{\omega}$, on the other hand, is given by

$$
\bar{\omega}=\frac{1}{R},
$$

as is seen from Eq. (82). As a result, the linear mechanical velocity, $\bar{v}=\bar{\omega} R$, is

$$
\bar{v}=1
$$


which coincides with the velocity of light. This is quite natural for a massless fluid, according to the discussion at the end of Sec. 5 in [18].

\section{Nonextremal shell}

For a nonextremal shell, the situation is what one expects upon imposing a reasonable regularity condition. We will see this now.

For nonextremal rotating shells, from Eq. (59) of [13] the thermodynamic angular velocity $\Omega$ is equal to

$$
\Omega=\frac{r_{-}}{r_{+} R \sqrt{\left(1-\frac{r_{+}^{2}}{R^{2}}\right)\left(1-\frac{r_{-}^{2}}{R^{2}}\right)}}\left[g\left(r_{+}, r_{-}\right)-\frac{r_{+}^{2}}{R^{2}}\right]
$$

where again $g\left(r_{+}, r_{-}\right)$is an arbitrary function of the gravitational and Cauchy radii, $r_{+}$and $r_{-}$, respectively. Now, if (i) the black hole limit $R \rightarrow r_{+}$is reachable and, (ii) we want to have $\Omega$ finite on the horizon, these conditions select the choice $g=1$, and hence

$$
\Omega=\frac{r_{-}}{r_{+} R} \sqrt{\frac{R^{2}-r_{+}^{2}}{R^{2}-r_{-}^{2}}}
$$

Then, $\Omega \sim \sqrt{R-r_{+}} \rightarrow 0$ in this limit.

The mechanical angular velocity $\bar{\omega}$ was obtained in Eq. (39) of [18] from matching conditions, namely,

$$
\bar{\omega}=\frac{r_{-}}{r_{+} R} \sqrt{\frac{R^{2}-r_{+}^{2}}{R^{2}-r_{-}^{2}}} .
$$

As we can see, in general, i.e., without imposing any condition, $\Omega$ given in Eq. (80) is different from $\bar{\omega}$ given in Eq. (82), $\Omega \neq \bar{\omega}$, so thermodynamic and mechanical angular velocities do not coincide. The same applies for the corresponding linear velocities, i.e., $v=\Omega R$ and $\bar{v}=$ $\bar{\omega} R$, do not coincide in general. However, for the choice $g=1$, one gets Eq. (81) that ensures the black hole limit, and both Eq. (81) and Eq. (82) coincide in this case, i.e., $\Omega=\bar{\omega}$.

To some extent, the situation resembles that with the temperature. Then, $T_{0} \neq T_{H}$ in general, where $T_{0}$ is the temperature of the shell measured at $r_{0}$ in the AdS case and $T_{H}$ is the Hawking temperature of a black hole with the same mass and other parameters. However, if the black hole limit is to be possible, we must take $T_{0}=T_{H}$ since otherwise an infinite backreaction would destroy the horizon, see [20] for more detailed discussion in the asymptotically flat case. Thus, in both cases, for the temperature and angular velocity, the existence of a well-defined black hole limit pushes forward the regularity conditions that make the choice of these quantities unambiguous.

\section{CONCLUSIONS}

We have investigated the thermodynamic entropy of an extremal rotating thin shell in the $(2+1)$-dimensional asymptotically AdS spacetime, where the exterior and interior of the shell were taken to be the BTZ spacetimes and the ground state AdS spacetime, respectively. The matching procedure of these two geometries is quite similar to the nonextremal shells, but nevertheless, the thermodynamic properties of the extremal shell have been shown to be quite different from those of the nonextremal shell.

For the extremal rotating shells, the thermodynamic state independent variables, namely, the local proper mass $M$ of the shell, the position $R$ of the shell, and the angular momentum $J$ of the shell, which appear in the first law of thermodynamics, are not independent but are constrained by the relation $J=M R$. Then, it was shown that the thermodynamic integrability condition does not restrict the form of the inverse temperature $\beta$ and rotational velocity $V$ equations of state of the shell, except that the product $\frac{\beta}{R}(1-V)$ must be solely a function of $r_{+}$. This also leads to the fact that the entropy $S$ is a function of the gravitational radius $r_{+}$alone, $S=S\left(r_{+}\right)$, or if one prefers, the gravitational area $A_{+}, S=S\left(A_{+}\right)$. To find the temperature distribution throughout the spacetime one must resort to the Tolman temperature formula.

We have considered two specific classes of equations of state for the temperature, one in which the local temperature at the shell is zero and the other in which the local temperature at the shell is nonzero and finite. We then took appropriately the extremal black hole limit and found that in both cases the extremal black hole entropy is $S=S\left(A_{+}\right)$, arguing convincingly that one should set $0 \leq S\left(A_{+}\right) \leq \frac{A_{+}}{4 G}$, i.e., the extremal black hole entropy has values in between zero and the maximum entropy, the Bekenstein-Hawking entropy $\frac{A_{+}}{4 G}$. Thus, rather than having just two entropies for the extremal black holes, as previous results have debated, namely zero and $\frac{A_{+}}{4 G}$, we have shown here that the extremal BTZ black hole entropy may have a continuous range of entropies, limited by precisely those two entropies. Surely, the entropy that a particular black hole picks must depend on past processes, notably on how it was formed. In the gravitational collapse of a shell to form an extremal black hole, the entropy could depend on the equation of state the shell, in the case of extremal black hole pair creation, the entropy could depend on the system initial conditions.

One can try to explain how the entropy of an extremal black hole is ambiguous and can take a range of values, $S=S\left(A_{+}\right)$. The no hair theorems for the the nonextremal BTZ black hole state that the final dynamical classical black hole is characterized only by its horizon radius $r_{+}$and Cauchy radius $r_{-}$(or by the mass $m$ and angular momentum $\mathcal{J}$ ), and for the extremal black hole only by $r_{+}$, as $r_{+}=r_{-}$(or equivalently by the mass $m$, as $\mathcal{J}=m \ell$ ). In the nonextremal case, the entropy is 
given in Eq. (2), $S=\frac{A_{+}}{4 G}$, and is a quantum, i.e., nonclassical, quantity at its very heart, since the area, or perimeter in the $(2+1)$ case, $A_{+}$is measured in terms of Planck areas $1 / G$ (recall the Planck constant is put to unity). Remarkably, this result is saying that the black hole entropy, the variable that characterizes the macroscopic quantum state, is characterized only by $A_{+}$which itself is a function only of the variables of the no hair theorems, namely, $r_{+}$and $r_{-}($or $m$ and $\mathcal{J})$. For the extremal case the situation is different. Although the area $A_{+}$still appears in the entropy, the entropy can be now a general function of the area itself, $S\left(A_{+}\right)$, and does not need to be just proportional to it. Thus, somehow the entropy for the extremal case has more freedom, it can depend on the initial object states that will form the extremal black hole, as for instance on the equation of state of the matter used to form the extremal black hole. At present, the origin of such an unusual behavior in the extremal case, that the entropy depends on the initial state of the system, is unknown, and a detailed elucidation of this issue would be of significant physical interest. Surely, fundamental theories to describe the entropy have to take this into account.

Two further important and interesting features have arisen in our analysis. One feature that came about in analyzing the shell with zero temperature is remarkable and should be mentioned. In this case we have found a relation between the limits $T \rightarrow 0$ and $V \rightarrow 1$, i.e., between the the impossibility of reaching absolute zero as the third law of thermodynamics states, and the impossibility for a massive body to reach the velocity of light. Another interesting feature that our considerations revealed and that deserves further analysis in a wider context of gravitational thermodynamics, not only for the $(2+1)$ dimensional spacetimes, is the relationship between two angular, or between two linear, velocities, namely, the mechanical velocity $\bar{v}$ and the thermodynamic velocity $V$. For the extremal rotating shells that we have studied this is especially pronounced since $\bar{v}=1$, while the thermodynamic velocity $V$ can take any value equal to or less than 1.

Appendix A: Tolman temperature and inverse temperature formulas for a spherical shell: General discussion, and the extremal and nonextremal cases

\section{Tolman inverse temperature formula for a spherical shell: The general formula}

The Tolman temperature formula states that the coordinate dependence of the temperature in a static background with radial coordinate $r$ is

$$
T=\frac{T_{0}}{k(r)}
$$

where $T_{0}$ depends not on $r$ but on some possible parameters related to the system, $T$ depends on $r$ through $k(r)$ only and on these possible parameters of the system, and $k(r)$ is the redshift function that depends essentially on $r$ but also can possibly depend on the other possible parameters. For the inverse temperatures $\beta$ and $b$, with $\beta=1 / T, b=1 / T_{0}$, formula (A1) turns into

$$
\beta=b k(r) .
$$

We will work with the inverse temperatures and the formula Eq. (A2). By inverting it, we can always revert to the relation between the temperatures themselves, Eq. (A1).

We start with a general nonextremal rotating BTZ spacetime in which a shell, or ring, is immersed at radius $R$. The discussion is quite general and, with small modifications, also holds for spacetimes with a spherical shell in other dimensions. For such a spacetime, the gravitational radius $r_{+}$, the Cauchy radius $r_{-}$, and the radius of the shell $R$ are the parameters that characterize the system. Thus, in general, for a nonextremal rotating BTZ spacetime with a shell at radius $R$, the Tolman general formula Eq. (A2) reads in this case

$$
\beta\left(r_{+}, r_{-}, R, r\right)=b\left(r_{+}, r_{-}, R\right) k\left(r_{+}, r_{-}, r\right),
$$

where now $k\left(r_{+}, r_{-}, r\right)$ for the nonextremal shell can be written explicitly as

$$
k\left(r_{+}, r_{-}, r\right)=\frac{r}{\ell} \sqrt{\left(1-\frac{r_{+}^{2}}{r^{2}}\right)\left(1-\frac{r_{-}^{2}}{r^{2}}\right)} .
$$

For a given set of the parameters $\left(r_{+}, r_{-}, R\right)$ we can consider the Tolman inverse temperature formula Eq. (A3) which gives the inverse temperature $\beta$ at a coordinate $r$ knowing the inverse temperature $b$ at the radius where $k=1$. I.e., $\beta$ is the inverse temperature at $r$, blueshifted or redshifted with factor $k$ from the inverse temperature $b$ at the position where $k=1$. One can then change the set $\left(r_{+}, r_{-}, R\right)$ and consider again the Tolman formula for the inverse temperature at $r$ for this new setting.

\section{Tolman inverse temperature formula for a spherical shell: The extremal case}

One can now treat the ab initio extremal case. For that, we take the extremal limit of the nonextremal shell, $r_{+} \rightarrow r_{-}$. Then Eq. (A3) gives in this limit

$$
\beta\left(r_{+}, R, r\right)=b\left(r_{+}, R\right) k\left(r_{+}, r\right),
$$

where now from Eq. (A4) one has $k\left(r_{+}, r\right)=\frac{r}{\ell}\left(1-\frac{r_{+}^{2}}{r^{2}}\right)$. As before, the function $b$ can be interpreted as the inverse temperature of the shell at the position $r_{0}$, where $k\left(r_{+}, r_{0}\right)=1$ (see also Eq. (13)). At the shell, $r=R$, the Tolman formula is

$$
\beta\left(r_{+}, R\right)=b\left(r_{+}, R\right) k\left(r_{+}, R\right),
$$


where

$$
k\left(r_{+}, R\right)=\frac{R}{\ell}\left(1-\frac{r_{+}^{2}}{R^{2}}\right) .
$$

There is no independent thermodynamic integrability condition for $\beta$ or $b$ and so the general equation for the thermodynamic shell is $b=b\left(r_{+}, R\right)$.

One could expect that the dependence on $R$ could be dropped, i.e., $b=b\left(r_{+}\right)$, which would be the case if one took directly the limit to an extremal case from a nonextremal case. But for an ab initio extremal shell, this does not happen, and one has indeed $b=b\left(r_{+}, R\right)$. This is the reason why extremal shells are different thermodynamic systems from nonextremal shells and extremal black holes are different thermodynamic systems from nonextremal black holes. See also Eqs. (44) and (46) in the main text.

In the above discussion, we implied that $R>r_{+}$, the quantity $b$ being finite. If a black hole limit is allowed, there are restrictions that force us to take $b=\infty$, see Secs. IV and V, and Tables I and II.

\section{Tolman inverse temperature formula for a spherical shell: The nonextremal case}

One can show that in the nonextremal case Eq. (A3) simplifies to

$$
\beta\left(r_{+}, r_{-}, R, r\right)=b\left(r_{+}, r_{-}\right) k\left(r_{+}, r_{-}, r\right),
$$

i.e., the $R$ dependence in $b\left(r_{+}, r_{-}, R\right)$ drops, and one gets instead simply $b\left(r_{+}, r_{-}\right)$, with $k\left(r_{+}, r_{-}, r\right)$ still being given by Eq. (A4).

Indeed, at $r=R$, the Tolman formula (A3) is $\beta\left(r_{+}, r_{-}, R, r=R\right)=b\left(r_{+}, r_{-}, R\right) k\left(r_{+}, r_{-}, r=\right.$ $R)$, i.e., $\beta\left(r_{+}, r_{-}, R\right)=b\left(r_{+}, r_{-}, R\right) k\left(r_{+}, r_{-}, R\right)$ with $k\left(r_{+}, r_{-}, R\right)=\frac{R}{\ell} \sqrt{\left(1-\frac{r_{+}^{2}}{R^{2}}\right)\left(1-\frac{r_{-}^{2}}{R^{2}}\right)}$. This is the Tolman inverse temperature expression at the shell radius $R$. If the shell happens to be at $R=R_{0}$, where $R_{0}$ is the radius at which $k\left(r_{+}, r_{-}, R_{0}\right)=1$, then at $R_{0}$, $\beta\left(r_{+}, r_{-}, R_{0}\right)=b\left(r_{+}, r_{-}, R_{0}\right)$.

Now, it is astonishing that the thermodynamic integrability conditions for the nonextremal BTZ shell case (this feature also holds for shells in other dimensions) give an equation which is a particular case of $\beta\left(r_{+}, r_{-}, R\right)=$ $b\left(r_{+}, r_{-}, R\right) k\left(r_{+}, r_{-}, R\right)$. Indeed, for the nonextremal rotating shells with two gravitational radii, $r_{+}$and $r_{-}$ $\left(r_{+}>r_{-}\right)$, the integrability conditions obtained from the first law of thermodynamics yield that the local inverse temperature equation of state of the shell [13] is

$$
\beta\left(r_{+}, r_{-}, R\right)=b\left(r_{+}, r_{-}\right) k\left(r_{+}, r_{-}, R\right),
$$

where $b\left(r_{+}, r_{-}\right)$is an arbitrary function of $r_{+}$and $r_{-}$and depends on the matter of the shell, and again $k$ is given by

$$
k\left(r_{+}, r_{-}, R\right)=\frac{R}{\ell} \sqrt{\left(1-\frac{r_{+}^{2}}{R^{2}}\right)\left(1-\frac{r_{-}^{2}}{R^{2}}\right)},
$$

which represents the gravitational redshift factor in the nonextremal BTZ spacetime. Note that here, from the integrability conditions, one finds $b=b\left(r_{+}, r_{-}\right)$, see Eq. (A9), whereas directly from Tolman formula, one has $b=b\left(r_{+}, r_{-}, R\right)$. Thus, thermodynamics, through its integrability conditions, restricts the parameter space for $b$. Equation (A9) is still the Tolman temperature equation for the shell at the shell, but a restricted form. Thus, by continuity, one also recovers the Tolman temperature formula for the whole spacetime displayed in Eq. (A8)

Note again that the extremal limit of Eq. (A8), i.e., of a nonextremal shell, is $\beta\left(r_{+}, R, r\right)=b\left(r_{+}\right) k\left(r_{+}, r\right)$, a particular, restrictive, case of the general ab inito extremal shell Eq. (A5). For extremal shells, the correct expression is, of course, Eq. (A5).

\section{ACKNOWLEDGEMENTS}

We thank Fundação para a Ciência e Tecnologia (FCT), Portugal, for financial support through Grant No. UID/FIS/00099/2013. JPSL thanks Coordenação de Aperfeiçoamento do Pessoal de Nível Superior (CAPES), Brazil, for support within the Programa CSF-PVE, Grant No. 88887.068694/2014-00. MM thanks FCT, Portugal, for financial support through Grant No. SFRH/BPD/88299/2012. OBZ has been partially supported by the Kazan Federal University through a state grant for scientific activities.
[1] M. Bañados, C. Teitelboim, and J. Zanelli, "The black hole in three-dimensional space-time", Phys. Rev. Lett. 69, 1849 (1992).

[2] D. Birmingham, I. Sachs, and S. Sen, "Exact results for the BTZ black hole", Int. J. Mod. Phys. D 10, 833 (2001); arXiv:hep-th/0102155.

[3] C. Teitelboim, "Action and entropy of extreme and nonextreme black holes", Phys. Rev. D 51, 4315 (1995); hep-th/9410103.

[4] D. Birmingham, I. Sachs, and S. Sen, "Entropy of threedimensional black holes in string theory", Phys. Lett. B424, 275 (1998); arXiv:hep-th/9801019.

[5] O. B. Zaslavskii, "Thermodynamics of $2+1$ black holes", Classical Quantum Gravity 11, L33 (1994).

[6] J. D. Brown, J. Creighton, and R. B. Mann, "Temperature, energy, and heat capacity of asymptotically anti- 
de Sitter black holes", Phys. Rev. D 50, 6394 (1994); arXiv:gr-qc/9405007.

[7] S. Carlip, "The statistical mechanics of the $(2+1)$ dimensional black hole", Phys. Rev. D 51, 632 (1995); arXiv:gr-qc/9409052.

[8] S. Carlip, "The (2+1)-dimensional black hole", Classical Quantum Gravity 12, 2853 (1995); arXiv:gr-qc/9506079.

[9] A. Strominger, "Black hole entropy from near horizon microstates", J. High Energy Phys. 02 (1998) 009; arXiv:hep-th/9712251.

[10] S. Wang, S.-Q. Wu, F. Xie, and L. Dan, "The first law of thermodynamics of the $(2+1)$-dimensional BañadosTeitelboim-Zanelli black holes and Kerr-de Sitter spacetimes", Chin. Phys. Lett 23, 1096 (2006); arXiv:hepth/0601147.

[11] T. Azeyanagi, T. Nishioka, and T. Takayanagi, "Near extremal black hole entropy as entanglement entropy via $\mathrm{AdS}_{2} / \mathrm{CFT}_{1}$ ", Phys. Rev. D 77, 064005 (2008); arXiv:0710.2956 [hep-th].

[12] J. P. S. Lemos and G. M. Quinta, "Entropy of thin shells in a $(2+1)$-dimensional asymptotically AdS spacetime and the BTZ black hole limit", Phys. Rev. D 89, 084051 (2014); arXiv:1403.0579 [gr-qc].

[13] J. P. S. Lemos, F. J. Lopes, M. Minamitsuji, and J. V. Rocha, "Thermodynamics of rotating thin shells in the BTZ spacetime", Phys. Rev. D 92, 064012 (2015); arXiv:1508.03642 [hep-th].

[14] Y. Peleg and A. R. Steif, "Phase transition for gravitationally collapsing dust shells in $(2+1)$-dimensions", Phys. Rev. D 51, R3992 (1995); arXiv:gr-qc/9412023.

[15] J. Crisóstomo and R. Olea, "Hamiltonian treatment of the gravitational collapse of thin shells", Phys. Rev. D 69, 104023 (2004); arXiv:hep-th/0311054.

[16] R. B. Mann, J. J. Oh, and M. I. Park, "Role of angular momentum and cosmic censorship in the $(2+1)$ dimensional rotating shell collapse", Phys. Rev. D 79, 064005 (2009); arXiv:0812.2297 [hep-th].

[17] J. Kim and M. Porrati, "More on long string dynamics in gravity on $\mathrm{AdS}_{3}$ : Spinning strings and rotating BTZ black holes", Phys. Rev. D 91, 124061 (2015); arXiv:1503.06875 [hep-th].

[18] J. P. S. Lemos, F. J. Lopes, and M. Minamitsuji, "Rotating thin shells in $(2+1)$-dimensional asymptotically AdS spacetimes: Mechanical properties, Machian effects, and energy conditions", Int. J. Mod. Phys. D 24, 1542022 (2015); arXiv:1506.05454 [gr-qc].

[19] E. A. Martinez, "Fundamental thermodynamical equation of a self-gravitating system", Phys. Rev. D 53, 7062 (1996); arXiv:gr-qc/9601037.

[20] J. P. S. Lemos, G. M. Quinta, and O. B. Zaslavskii, "Entropy of a self-gravitating electrically charged thin shell and the black hole limit", Phys. Rev. D 91, 104027 (2015); arXiv:1503.00018 [hep-th].

[21] J. P. S. Lemos, G. M. Quinta, and O. B. Zaslavskii, "Entropy of an extremal electrically charged thin shell and the extremal black hole", Phys. Lett. B 750, 306 (2015); arXiv:1505.05875 [hep-th].

[22] J. P. S. Lemos, G. M. Quinta, and O. B. Zaslavskii, "Entropy of extremal black holes: Horizon limits through charged thin shells in a unified approach", Phys. Rev. D
93, 084008 (2016); arXiv:1603.01628.

[23] J. P. S. Lemos and O. B. Zaslavskii, "Entropy of quasiblack holes", Phys. Rev. D 81, 064012 (2010); arXiv:0904.1741 [gr-qc].

[24] J. P. S. Lemos and O. B. Zaslavskii, "Entropy of extremal black holes from entropy of quasiblack holes", Phys. Lett. B 695, 37 (2011); arXiv:1011.2768 [gr-qc].

[25] F. Pretorius, D. Vollick, and W. Israel, "An operational approach to black hole entropy", Phys. Rev. D 57, 6311 (1998); arXiv:gr-qc/9712085.

[26] S. W. Hawking, G. T. Horowitz, and S. F. Ross, "Entropy, area, and black hole pairs", Phys. Rev. D 51, 4302 (1995); arXiv:gr-qc/9409013.

[27] A. Strominger and C. Vafa, "Microscopic origin of the Bekenstein-Hawking entropy", Phys. Lett. B 379, 99 (1996); arXiv:hep-th/9601029.

[28] A. Sen, "Microscopic and macroscopic entropy of extremal black holes in string theory", Gen. Relativ. Gravit. 46, 1711 (2014); arXiv:1402.0109 [hep-th].

[29] G. W. Gibbons and R. E. Kallosh, "Topology, entropy and Witten index of dilaton black holes", Phys. Rev. D 51, 2839 (1995); arXiv:hep-th/9407118.

[30] A. Ghosh and P. Mitra, "Understanding the area proposal for extremal black hole entropy", Phys. Rev. Lett. 78, 1858 (1997); arXiv:hep-th/9609006.

[31] S. Hod, "Evidence for a null entropy of extremal black holes", Phys. Rev. D 61, 084018 (2000); arXiv:grqc/0004003.

[32] S. M. Carroll, M. C. Johnson, and L. Randall, "Extremal limits and black hole entropy", J. High Energy Phys. 11 (2009) 109; arXiv:0901.0931 [hep-th].

[33] A. Edery and B. Constantineau, "Extremal black holes, gravitational entropy and nonstationary metric fields", Classical Quantum Gravity 28, 045003 (2011); arXiv:1010.5844 [gr-qc].

[34] A. Ghosh and P. Mitra, "Entropy for extremal ReissnerNordström black holes", Phys. Lett. B 357, 295 (1995); arXiv:hep-th/9411128.

[35] R. B. Mann and S. N. Solodukhin, "Universality of quantum entropy for extreme black holes", Nucl. Phys. B 523, 293 (1998); arXiv:hep-th/9709064.

[36] C. Kiefer and J. Louko, "Hamiltonian evolution and quantization for extremal black holes", Annalen Phys. 8, 67 (1999); arXiv:gr-qc/9809005.

[37] G. A. S. Dias and J. P. S. Lemos, "Hamiltonian thermodynamics of d-dimensional ( $d \geq 4$ ) Reissner-Nordström anti-de Sitter black holes with spherical, planar, and hyperbolic topology", Phys. Rev. D 79, 044013 (2009); arXiv:0901.0278 [gr-qc].

[38] O. B. Zaslavskii, "Entropy of quantum fields for nonextreme black holes in the extreme limit", Phys. Rev. D 57, 6265 (1998); arXiv:gr-qc/9708027.

[39] J. D. Bekenstein, "Black holes and entropy", Phys. Rev. D 7, 2333 (1973).

[40] J. M. Bardeen, B. Carter, and S. W. Hawking, "The four laws of black hole mechanics", Commun. Math. Phys. 31, 161 (1973).

[41] S. W. Hawking, "Particle creation by black holes", Commun. Math. Phys. 43, 199 (1975). 